Gut, 1986, 27, 1096-1100

Case reports

\title{
Ulcerative colitis complicated by Budd-Chiari syndrome
}

\author{
I M CHESNER, S MULLER, AND J NEWMAN \\ From the Metabolic Unit and Histopathology Department, East Birmingham Hospital, Bordesley Green East, \\ Birmingham
}

SUMMARY We present two cases of ulcerative colitis associated with a chronic pattern of hepatic vein thrombosis (Budd-Chiari syndrome). Progression of the hepatic disorder appeared to be unrelated to the activity of the colitis. Liver function tests were normal until a late stage in the disease except for a high serum alkaline phosphate and a low serum albumin. This combination of abnormalities together with ascites merit further investigation and should raise the suspicion of hepatic vein thrombosis complicating ulcerative colitis.

Patients with ulcerative colitis have a greater tendency to venous thrombosis presenting both clinically ${ }^{1-5}$ and found incidentally at necropsy. The commonest sites for venous thrombosis are the pulmonary vasculature and the deep veins of the legs. In one necropsy study $39 \%$ of patients with ulcerative colitis were found on detailed examination to have evidence of venous thrombosis. ${ }^{6} \mathrm{Hepa}-$ tic vein thrombosis, however, complicating ulcerative colitis, although recognised, is rare. In his large review of 164 necropsy confirmed cases of hepatic vein thrombosis, Parker ${ }^{7}$ reports only two cases in his personal experience and two others in the literature ${ }^{89}$ with underlying ulcerative colitis. We report two cases of established ulcerative colitis complicated by hepatic vein thrombosis which differ in certain respects from the previous reports.

\section{Case 1}

A 54 year old woman presented with a two week history of pain in her right leg associated with malaise, vomiting, and diarrhoea. The only previous history of significance was of schizophrenia diagnosed nine years previously.

On examination she was clinically anaemic with a

Address for correspondence: Dr J Newman, Department of Histopathology, East Birmingham Hospital, Bordesley Green East, Birmingham B9 5ST. Received for publication 13 December 1985 vasculitic skin rash and the appearances of resolving cellulitis over her right calf. There were no abdominal masses and the liver was not enlarged. Sigmoidoscopy showed a friable rectal mucosa with contact bleeding and moderate amounts of mucus and blood on the surface. Investigation revealed $\mathrm{Hb}$ $8.9 \mathrm{~g} / \mathrm{l}, \mathrm{MCV} 85 \mathrm{fl}$, electrolytes and liver function tests were normal apart from serum albumin $29 \mathrm{~g} / \mathrm{l}$. Orosomucoid was raised at $3 \cdot 29 \mathrm{~g} / \mathrm{l}$. She had a positive ANF 1:400 but no DNA binding; immune complexes by platelet aggregation were present to a titre of 1:60. Stool culture was negative as was microscopy for ova, cysts, and parasites. Rectal biopsy showed an acute inflammatory infiltrate in the lamina propria with occasional crypt abscesses, goblet cell depletion and branched glands, changes compatible with ulcerative colitis. A barium enema confirmed left sided colitis. She was transfused and given intravenous hydrocortisone and oral sulphasalazine.

Response to treatment was slow. Her diarrhoea improved, but after two weeks she developed abdominal swelling and was noted to be drowsy. On examination she was not icteric, but had moderate ascites with no abdominal tenderness. Liver function tests still showed a low serum albumin $24 \mathrm{~g} / \mathrm{l}$ but were otherwise normal. Ascitic fluid had a protein content of $14.5 \mathrm{~g} / \mathrm{l}$. Liver ultrasound showed multiple echogenic areas and an isotope scan showed 
poor uptake by the right lobe. Liver biopsy done at this time showed a minimal portal lymphocytic infiltrate. There was one focus of centrilobular congestion, with slight sinusoidal dilatation, but there was no evidence of fibrosis, and these changes were regarded as non-specific. her ascites settled on treatment with diuretics and liver function tests did not change.

Five months later she was readmitted with increasing diarrhoea and anaemia (Hb $9.2 \mathrm{~g} / \mathrm{l})$. Sigmoidoscopy revealed an acute colitis and this responded to topical steroid enemas. Liver function tests were normal apart from a low albumin $(27 \mathrm{~g} / \mathrm{l})$. Seven months later she developed left calf pain. On examination she was pale, emaciated, with an erythematous rash over her thighs and buttocks and an ulcer on her left calf. Additionally she had developed arthralgia of her knees and elbows. New findings on examination were $6 \mathrm{~cm}$ hepatomegaly and moderate ascites. Biopsy of the leg ulcer showed changes compatible with pyoderma gangrenosum. Investigations at this time revealed $\mathrm{Hb} 9.7$ $\mathrm{g} / \mathrm{l}$, albumin $15 \mathrm{~g} / \mathrm{l}$. Liver function tests were normal except for a raised alkaline phosphatase $(567 \mathrm{IU} / \mathrm{l})$ (normal less than $350 \mathrm{IU} / \mathrm{l}$ ). Rectal biopsy showed quiescent colitis. She was treated with parenteral hydrocortisone, but over the next six weeks her liver function tests gradually deteriorated, bilirubin rising to $162 \mu \mathrm{mol} / \mathrm{l}$, alkaline phosphatase rising to 877 IU/l, aspartate transaminase $370 \mathrm{IU} / \mathrm{l}$, Gamma GT $163 \mathrm{IU} / \mathrm{l}, \mathrm{LDH} 870 \mathrm{IU} / \mathrm{l}$. In spite of a liver failure regime, she developed encephalopathy and died.

\section{NECROPSY FINDINGS}

The salient findings were confined to the abdominal cavity where there were 41 straw coloured ascites. The liver $(1120 \mathrm{~g})$ had a smooth capsule and a dusky red colour on its cut surface. There was intense sinusoidal congestion together with occasional paler and more normal looking areas, mainly around the hilum. Recent thrombi were present in many of the small hepatic venous radicles and in the main hepatic veins. The portal vein and its branches were patent, as was the inferior vena cava.

Histology showed that the majority of the hepatic venous branches were occluded by thrombi in various stages of organisation, fibrous obliteration, and recanalisation. The parenchyma displayed a variable degre of centrilobular congestion, necrosis, and minimal patchy central-central bridging with fibrosis (Fig. 1).

The proximal colon appeared normal, whilst the

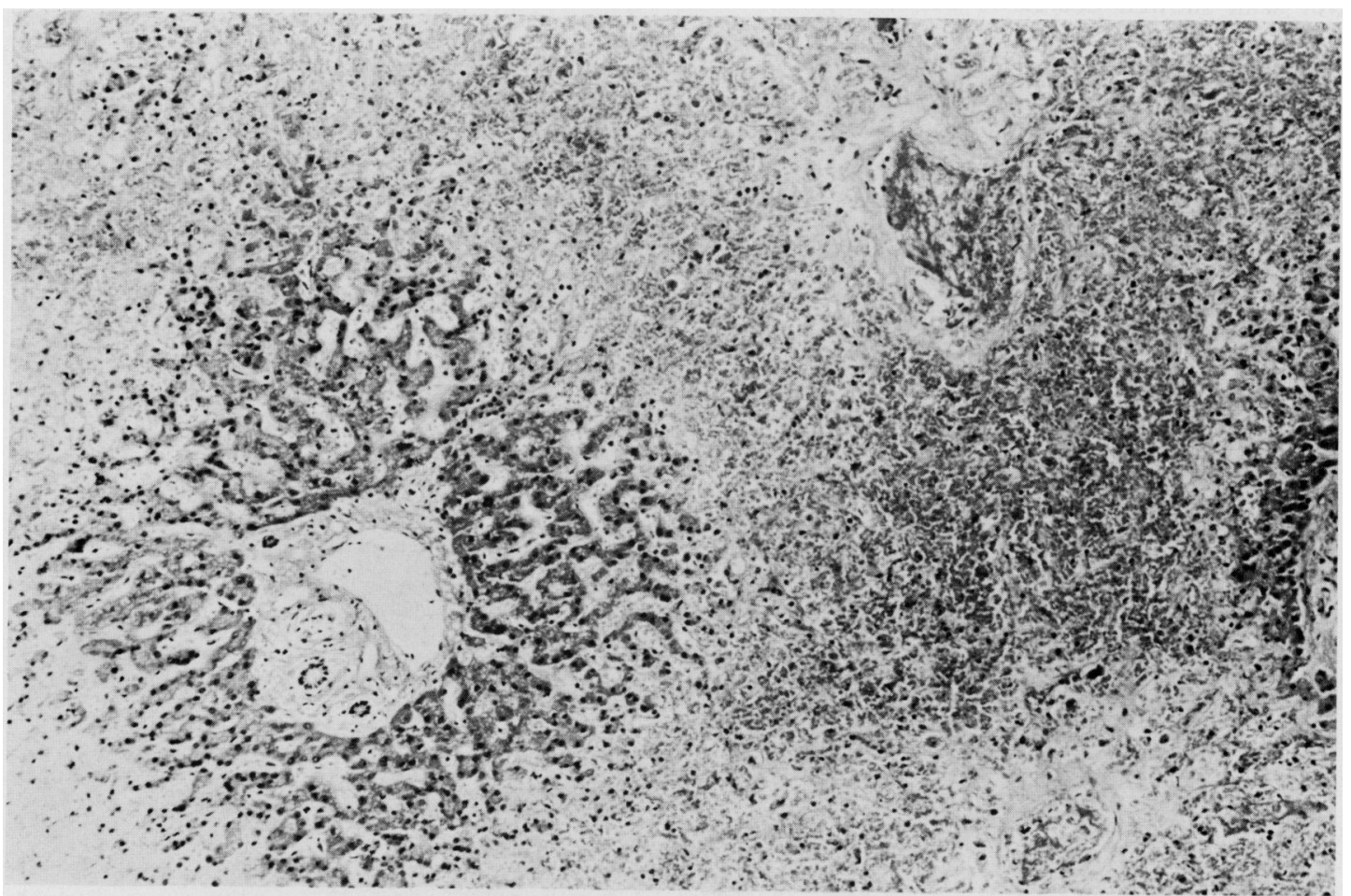

Fig. 1 Portal tract (left) with a rim of viable hepatocytes; central veins (right) occluded by fibrin thrombi, with surrounding haemorrhage and necrosis. $(H \& E \times 68)$. 
distal $45 \mathrm{~cm}$ was rigid, fixed, and shortened. The distal mucosa was diffusely and continuously abnormal with map-like areas of flattened mucosa merging with polypoid areas.

Histological examination showed acute on chronic change, with sharply demarcated ulcers separated by mucosa with mild diffuse chronic inflammation as well as occasional crypt abscesses. The glands displayed architectural abnormalities including branching and focal Paneth cell metaplasia.

\section{Case 2}

A 35 year old male engineer presented in 1976 with short history of bloody diarrhoea and weight loss. Sigmoidoscopy showed changes of acute ulcerative colitis, confirmed on rectal biopsy. Barium enema revealed total colitis with backwash ileitis. He was treated initially with steroids and sulphasalazine and remained well controlled for six years on maintenance sulphasalazine. In 1982 he presented with lower abdominal pain, and on examination had a tender, enlarged liver, and moderate ascites. His liver function tests showed a raised alkaline phosphatase $895 \mathrm{IU} / \mathrm{l}$ and a low albumin $28 \mathrm{~g} / \mathrm{l}$. Serum transaminases and bilirubin were normal. Isotope liver scan showed decreased hepatic uptake with increased uptake by lung and bone marrow suggesting poor liver perfusion. A barium enema and colonoscopy revealed total ulcerative colitis with inflammatory polyps. Liver biopsy showed focal portal tract expansion and fibrosis. There was, however, also focal centrilobular sinusoidal dilatation, with liver cell atrophy and reticulin condensation, features suggestive of Budd-Chiari syndrome. He was treated conservatively with diuretics and subcutaneous heparin and improved over a two month period. For a further two years his colitis was well controlled on sulphasalazine alone and his liver function tests showed gradual improvement although serum alkaline phosphatase remained high. Colonoscopy in January 1985 showed quiescent left sided colitis with active disease in the transverse, right colon and caecum, confirmed on biopsy. One month later he developed severe right hypochondrial pain and hepatic tenderness. On examination he was not icteric or encephalopathic. There was $5 \mathrm{~cm}$ tender hepatomegaly and moderate ascites with a protein content of $17 \mathrm{~g} / \mathrm{l}$. Liver function tests showed raised alkaline phosphatase $694 \mathrm{IU} / \mathrm{l}$, AST $121 \mathrm{IU} / \mathrm{l}$, bilirubin $27 \mu \mathrm{mol} / \mathrm{l}$, albumin $22 \mathrm{~g} / \mathrm{l}$ and a prolonged prothrombin time. Isotope liver scan showed selective uptake by the caudate lobe with minimal uptake in the remainder of the right lobe of the

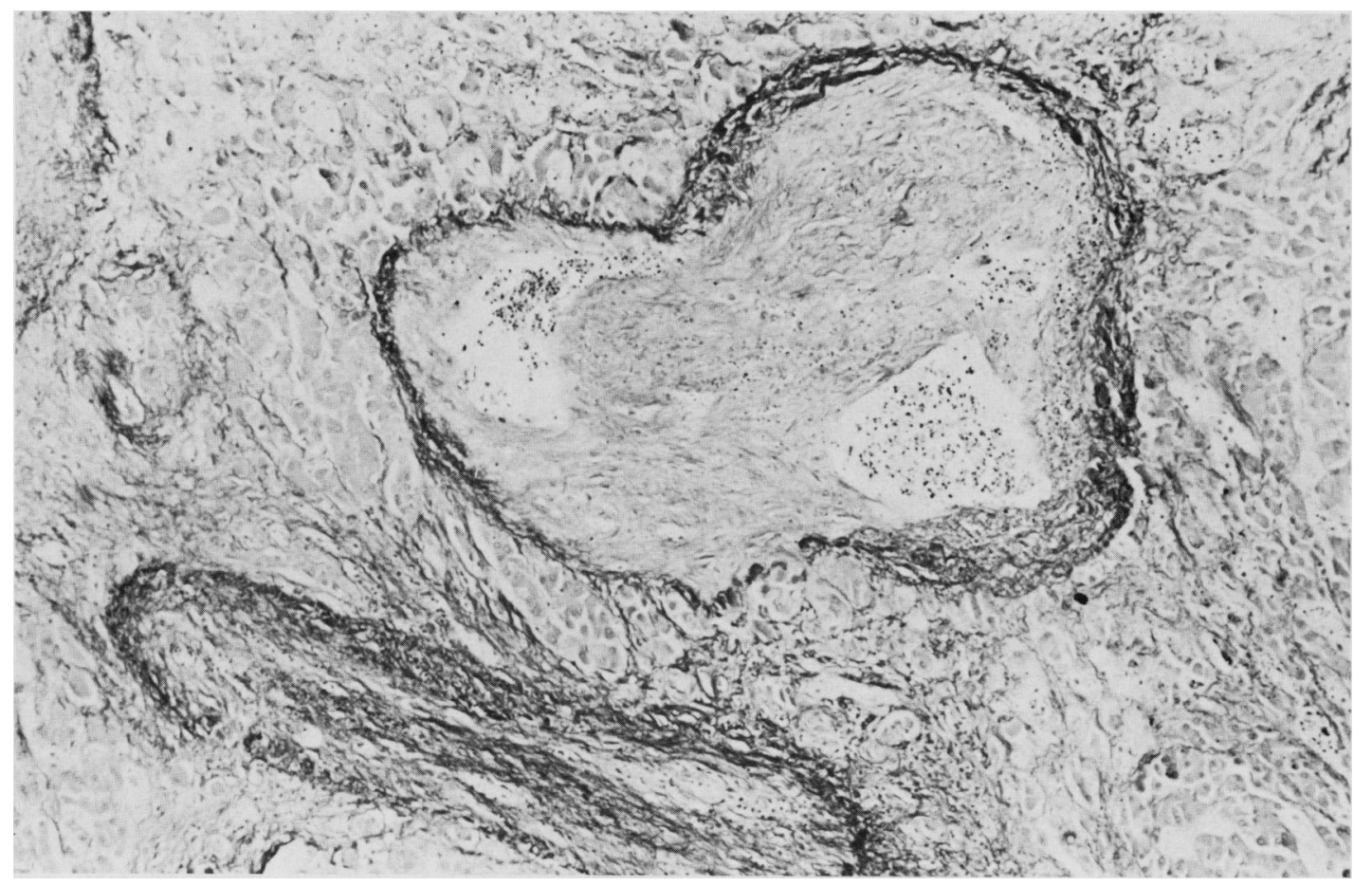

Fig. 2 Partly occluded and recanalised hepatic vein. (Elastic van Gieson $\times 68$ ). 
liver, compatible with Budd-Chiari syndrome. He was given intravenous heparin but after an initial improvement deteriorated with progressive encephalopathy. Treatment of his liver failure produced no improvement and he died five days later.

\section{NECROPSY FINDINGS}

The peritoneal cavity contained three litres of straw coloured fluid. The liver $(1500 \mathrm{~g})$ had a shrunken and wrinkled capsule, without obvious caudate lobe enlargement. The cut surface showed a pattern of chronic venous congestion, together with larger irregular haemorrhagic zones. The hepatic venous ostia were small, many contained slightly protuberant fleshy yellow nodules which were in continuity with hepatic venous tributaries containing recent red thrombus. The inferior vena cava itself was normal. Both the splenic and portal veins contained fresh thrombus, which extended into large intrahepatic portal venous branches.

Histological examination revealed recent fibrin and platelet rich thrombi occluding many of the hepatic venous tributaries; the yellow nodules representing older partially recanalised thrombi. Similar lesions were present in many of the smaller intrahepatic venous branches but these, however, showed a greater degree of recanalisation (Fig. 2). Several portal venous branches were occluded by recent thrombus, some showing early organisation. Hepatic parenchymal changes were variable and patchy. In some areas there was gross centrilobular congestion and necrosis, with central-central linking and fibrosis, viable hepatocytes being present only in periportal zones - that is, 'reverse lobulation'. Other areas, however, showed only minimal central congestion.

The colon was dilated and shortened. The mucosa was flat and atrophic; pseudopolyps were noted in the transverse colon. Histological examination confirmed chronic predominantly quiescent ulcerative colitis with glandular distortion, branching, and atrophy, but only occasional crypt abscesses.

\section{Discussion}

Well recognised hepatic complications of ulcerative colitis include fatty change, pericholangitis, sclerosing cholangitis, chronic active hepatitis and rarely, cirrhosis. ${ }^{10}$ Hepatic vein thrombosis has been documented as an acute terminal event in ulcerative colitis,${ }^{89}$ however, clinical evidence of long standing chronic hepatic venous occlusion is not described in association with ulcerative colitis. Parker's series of 164 cases described only two patients with hepatic vein thrombosis associated with ulcerative colitis; both of these were incidental findings at necropsy and clinical details were not given. Symptomatic cases with a well documented acute history and autopsy confirmation were described by Kelsey ${ }^{8}$ and Jorgensen. ${ }^{9}$ Both of our cases clearly show that as well as acute thrombosis there was evidence of chronic hepatic venous occlusion, with histological features of recanalisation, indicating that the process had been evolving for some time. This pattern of thrombosis, with lesions of varying age as judged histologically, is typical of Budd-Chiari syndrome.

Thirty nine per cent of Graef's series had evidence of venous thrombosis ${ }^{6}$ compared with $14 \%$ of controls. 'Visceral' venous thrombosis was noted in $16 \%$ although the exact sites were not further specified. In all of these cases the age of the thrombus varied, with $20 \%$ showing evidence of both recent and old thrombosis. Mechanisms subsequently postulated by $\mathrm{Lee}^{11}$ for this thrombotic tendency were a hypercoagulable state resulting from high fibrinogen levels, increased thromboplastin generation, and raised factor VIII activity. Lake $^{12}$ showed a qualitative platelet defect and reduced antithrombin III activity in patients with ulcerative colitis. In addition Talstad ${ }^{13}$ noted that over half his series of patients with moderate or severe ulcerative colitis had a persistent thrombocytosis $(>400000 / \mu \mathrm{l})$ with decreased platelet half life and increased turnover.

Terminally both our cases presented with classical signs and symptoms of hepatic vein thrombosis (abdominal pain, hepatomegaly and ascites). Earlier in the course of their disease, however, both had evidence of ascites with a low protein content, associated with a low serum albumin concentration and raised serum alkaline phosphatase. This pattern of liver function abnormality is not characteristic of any other disease known to complicate ulcerative colitis, and has not previously been documented in cases of hepatic vein thrombosis associated with ulcerative colitis. Liver function tests in hepatic vein thrombosis are variable ${ }^{14}$ there being no specific diagnostic pattern, although more severe disturbances are seen in acute hepatic vein thrombosis. The picture of chronic hepatic vein thrombosis is well illustrated by the two cases we have described.

Neither case showed evidence of a haematological disorder, malignancy, or any other disease with a recognised association with hepatic vein thrombosis. In both cases immediately before fulminant hepatic failure their colitis was asymptomatic. This chronic pattern of hepatic vein thrombosis in association with ulcerative colitis differs from the acute fulminant course previously described by Kelsey ${ }^{8}$ and Jorgensen. ${ }^{9}$ Progressive venous thrombosis occurred in spite of adequate control of colonic inflammation. Perhaps active therapy of the hypercoagulable 
state present in ulcerative colitis may be indicated in patients recognised to have the more chronic form of hepatic vein thrombosis.

The authors would like to thank Dr J Gearty, who carried out one of the post mortem examinations, Mr M J Chard for assistance with the photographs, and Dr P Asquith for his permission to report these cases.

\section{References}

1 Bargen JA, Barker MW. Extensive arterial and venous thrombosis complicating chronic ulcerative colitis. Arch Intern Med 1936; 58: 17-31.

2 Ricketts WE, Palmer WL. Complications of chronic non-specific ulcerative colitis. Gastroenterology 1946; 7: $55-66$.

3 Warren S, Sommers SC. Pathogenesis of ulcerative colitis. Am J Pathol 1949; 25: 657-79.

4 Aronson AR, Steinheber FU. Portal vein thrombosis in ulcerative colitis. NY State J Med 1971; 71: 2310-11.

5 Lam A, Borda IT, Inwood MJ, Thompson S. Coagulation studies in ulcerative colitis and Crohn's disease. Gastroenterology 1975; 68: (2) 245-51.
6 Graef V, Baggenstoss AH, Sauer WG, Spittell JA Jnr. Venous thrombosis occurring with non-specific ulcerative colitis. Arch Intern Med 1966; 117: 377-82.

7 Parker RGF. Occlusion of the hepatic veins in man. Medicine (Balt) 1959; 38: 369-402.

8 Kelsey MP, Comfort MW. Occlusion of the hepatic veins. A review of 20 cases. Arch Intern Med 1945; 75: 175-83.

9 Jorgensen L. Occlusion of the hepatic veins. Acta Pathol Microbiol Scand 1958; 44: 68-79.

10 Parker RGF, Kendall EJC. The liver in ulcerative colitis. Br Med J 1954; ii: 1030-2.

11 Lee LC, Spittell JA Jnr, Sauer WG, Owen CA Jnr, Thomson JH. Hypercoagulability associated with chronic ulcerative colitis-changes in blood coagulation factors. Gastroenterology 1968; 54: 76-85.

12 Lake AM, Stauffer JQ, Stuart MJ. Haemostatic alterations in inflammatory bowel disease. Response to therapy. Am J Dig 1978; 23: 897-902.

13 Talstad I, Rootwelt K, Gjone E. Thrombocytosis in ulcerative colitis and Crohn's disease. Scand J Gastroenterol 1973; 8: 135-8.

14 Powell-Jackson PR, Melia W, Canalese J, Pickford RB, Portman B, Williams R. Budd-Chiari syndrome: clinical patterns and therapy. $Q J$ Med 1982; 5: 179-88. 